\title{
Assessing Calcification Onset in Aortic Valve Interstitial Cells
}

Khan K, Yu B, Al-Kindi H, Cecere R and Schwertani A*

Cardiology and Cardiac Surgery, McGill University Health Centre, Montreal, Quebec, Canada

\begin{abstract}
Aortic valve stenosis (AVS) is one of the most common heart valve diseases, and surgical intervention remains the only viable treatment option. Thus, there is a need for novel and innovative treatments. One approach is to study the biological pathogenesis of this disease in hopes of finding new targets for drug therapy. Although this may be a viable option, it faces some methodological concerns. Many studies attempted to address the underlying mechanisms of this disease using aortic valves from bovine models. Although these may be viable models in certain diseased conditions, this may not be the case when studying calcification in AVS. Thus, the aim of our study was to assess the significance of drawing conclusions from bovine models to humans in the context of AVS, and investigate the role of alkaline phosphatase (ALP), an enzyme that increases calcium mineralization and deposition in aortic valve calcification. We also wanted to identify any differences in calcification when using different osteogenic media.

We used human and bovine valve interstitial cells (HAVICs and BAVICs, respectively), which are the most commonly used when study calcification in AVS, and cultured them in osteogenic media or DMEM as control media. We found that ALP activity differs widely between the two models, with bovine samples having approximately ten times more ALP activity. Our data also suggests that the degree of calcification and ALP activity differs between the different osteogenic media used.
\end{abstract}

Careful consideration should be taken when experimenting with bovine valves and drawing conclusions to human AVS, as they may not exhibit the same mechanisms of action. Furthermore, it may be important to identify a single standard osteogenic medium to use when studying calcification in AVS.

Keywords: Aortic valve stenosis; Calcification; Human; Bovine; ALP

\section{Introduction}

Aortic valve stenosis (AVS) is a complex disease, characterized by the thickening of the aortic valve with significant fibrosis and/or calcification [1]. Short-term symptoms include shortness of breath and fainting, while more long-term symptoms include heart failure and inevitably, death [2]. Treatment for AVS remains non-existent, with surgical intervention being the only viable option for at-risk patients. Thus, there has been interest in finding innovative approaches to treating this perilous disease $[3,4]$.

The pathogenesis of AVS is an active process involving multiple cell types and complexed underlying mechanisms [5,6]. Upon differentiation of mesenchymal-like cells to chondrogenic cells, matrix vesicles are released, containing enzymes and other factors that lead to the onset of calcification [7]. One of these enzymes, alkaline phosphatase (ALP), is a metalloenzyme that has been shown to play a profound role in calcification onset by concentrating calcium [8], mineralizing hydroxyapatite crystals [8], and inactivating inhibitory polyphosphates [9]. In humans, there are four ALP isozymes: alkaline phosphatase, tissue-nonspecific isozyme (TNALP), intestinal-type alkaline phosphatase, placental-type alkaline phosphatase, and placental-like alkaline phosphatase [10]. Two isoforms of TNALP exist: bone-specific TNALP and liver-specific TNALP, both of which are abundantly found in the serum [10]. Within the context of vascular calcification, bone TNALP is highly expressed in calcifying vascular smooth muscle cells and is likely to be responsible for calcium deposition and AVS pathogenesis [10].

A multitude of studies draw conclusions regarding the pathogenesis of AVS in humans by utilizing aortic valve interstitial cells from bovine [11-13]. The ultimate goal of these studies is to identify mechanisms that will help us better understand calcification onset in humans. However, there is a need to directly compare these animal models to humans and assess whether or not it is plausible to make such a comparison. Therefore, the objectives of our study were to assess the onset of calcification and its related mechanisms in human and bovine aortic valve interstitial cells (HAVICs and BAVICs, respectively) cultured in either Dulbecco's Modified Eagle's Medium (DMEM) or osteogenic media (OSM).

\section{Materials and Methods}

\section{Isolation and culture of valve interstitial cells}

Primary HAVIC lines were generated as previously described $[14,15]$. Cultured HAVICs showed positive staining for alpha smooth muscle actin, indicating myofibroblast phenotype after 2 passages. HAVICs at passages 3 to 5 were used for all experiments. BAVICs were isolated using similar methodology.

\section{Inducing calcification in aortic valve interstitial cells}

HAVICs and BAVICs were plated in 24-well plates until 100\% confluent and incubated in different OSM for 7 and 12 days, respectively. Five types of OSM were used: full DMEM with the addition of $2 \mathrm{mM}$ Phosphate buffer, pH 7.4 (Os-P); 2 mM phosphate buffer, $\mathrm{pH}$ 7.4, with $0.2 \mathrm{mM}$ ascorbic acid (Os-PC); $2 \mathrm{mM}$ Phosphate buffer, $\mathrm{pH} 7.4$, with $0.2 \mathrm{mM}$ ascorbic acid and $1 \mathrm{ug} / \mathrm{mL}$ of insulin (Os-PCI); $10 \mathrm{mM}$

*Corresponding author: Adel Schwertani, Cardiology and Cardiac Surgery, McGill University Health Centre, Suite EM1.2224, 1001 Decarie Blvd, Montreal, Quebec H4A 3J1, Canada, Tel: 514934 1934/43841; E-mail: adel.schwertani@mcgill.ca

Received September 13, 2017; Accepted October 05, 2017; Published October 12, 2017

Citation: Khan K, Yu B, Al-Kindi H, Cecere R, Schwertani A (2017) Assessing Calcification Onset in Aortic Valve Interstitial Cells. Cardiovasc Pharm Open Access 6: 222. doi: 10.4172/2329-6607.1000222

Copyright: () 2017 Khan K, et al. This is an open-access article distributed under the terms of the Creative Commons Attribution License, which permits unrestricted use, distribution, and reproduction in any medium, provided the original author and source are credited. 
$\beta$-glycerol phosphate (Os-BP); or $10 \mathrm{mM} \beta$-Glycerol phosphate with $0.2 \mathrm{mM}$ ascorbic acid (Os-BPC).

\section{Assessment of ALP activity}

ALP Activity Colorimetric Assay Kit (BioVision) was used to asses ALP activity according to the manufacturer's instructions. Briefly, after treating cells with, EDTA and/or Lp(a) for 7 days (Athens Research and Technology Inc.), the culture medium was harvested and spun for 10 minutes at 12,000 g; supernatant was used for ALP activity assay. Cells were washed twice with PBS, then incubated with shaking for 10 minutes at room temperature in cell lysis buffer $(20 \mathrm{mM}$ Tris- $\mathrm{HCl}$ $\mathrm{pH}=8.0,150 \mathrm{mM} \mathrm{NaCl}, 0.2 \% \mathrm{NP} 40)$. Cell lysates were harvested and spun at $12,000 \mathrm{~g}$ for 5 minutes at $4^{\circ} \mathrm{C}$, supernatant was harvested for ALP activity assay.

\section{Assessment of calcification}

Alizarin Red S Staining Quantification Assay (AR-S) was used to assess calcium deposition and calcium nodule formation. Cells were plated in 24 -well plates until $100 \%$ confluent and treated with different OSM for 7 days. Briefly, cells were cultured in different OSM and fixed for 30 minutes using $4 \%$ buffered formaldehyde at room temperature. Cells were then washed 4 times with excessive $\mathrm{ddH}_{2} \mathrm{O}$, incubated with Alizarin Red S stain solution ( $40 \mathrm{mM}, \mathrm{pH} 4.2)$ for 20 minutes at room temperature, and again washed 4 times with excessive $\mathrm{ddH}_{2} \mathrm{O}$. Calcification nodules were observed under microscopy, and phase contrast images were taken with inverted microscopy. Similar assessment of calcification was performed in another set of HAVICs treated with the XAV939, the inhibitor of Wnts catenin-mediated transcription through tankyrase $1 / 2$.

\section{Assessment of cellular proliferation}

MTT (Thiazolyl Blue Tetrazolium Blue, Sigma) assay was used to assess cellular proliferation and is presented as relative proliferation rate. Briefly, HAVICs were treated with $1-20 \mu \mathrm{M}$ of XAV939 for 48 hours. MTT solution ( $5 \mathrm{mg} / \mathrm{ml}$ in DMEM) was added to cultured cells in 24-well plates, and incubated for $2-3$ hours at $37^{\circ} \mathrm{C}$. The purple precipitation was extracted using MTT solvent (isopropanol with 4 $\mathrm{mM} \mathrm{HCl}, 0.1 \%$ Nondet P-40), and measured at OD570 nm with 96well reading plate on Spectra Photometer (BioTek).

\section{Statistical analysis}

One-way ANOVA tests were used to assess ALP activity and AR-S quantification in the three lines of AVICs. Statistical analysis was performed using GraphPad Prism software version 6.0 (GraphPad Software Inc., La Jolla, CA, USA). P-values of $<0.05$ were considered to be statistically significant. All values are represented as mean \pm SEM.

\section{Results}

\section{Comparison of ALP activity in HAVICs and BAVICs}

HAVICs isolated from calcified valves had significantly greater ALP activity compared to those isolated from non-calcified valves $(\mathrm{P}<0.05)$. Incubation of HAVICs in OSM significantly increased ALP activity in cells from non-calcified and calcified valves $(\mathrm{P}<0.05)$. There was a significant difference in ALP activity detected in HAVICs compared to BAVICs when cultured in both DMEM or OSM. Indeed, BAVICS cultured in OSM had almost 10 times the amount of ALP compared to those of HAVICs cultured in the same medium $(\mathrm{P}<0.0001)$ (Table 1).

\section{Calcium deposition in HAVICs and BAVICs cultured in different OSM}

AR-S staining showed the presence of significant calcification in HAVICs cultured in Os-P, Os-PC, Os-PCI, Os-BP, Os-BPC compared to DMEM controls (Figure 1A and B). Similarly, BAVICs exhibited significant calcification when cultured in Os-P, Os-PC, Os-BP, Os-BPC compared to DMEM controls (Figure 2A and B). Os-BPC showed the most significant calcification among all media tested $(\mathrm{P}<0.0001)$. Os-PC was the least effective calcifying medium

\begin{tabular}{|c|c|c|c|c|}
\hline \multirow{2}{*}{} & \multicolumn{2}{|c|}{ DMEM } & \multicolumn{2}{c|}{ OSM } \\
\cline { 2 - 5 } & Mean & SEM & Mean & SEM \\
\hline $\begin{array}{c}\text { Non-calcified } \\
\text { HAVICs }\end{array}$ & $2.67^{\mathrm{a}}$ & 0.26 & $3.19^{\mathrm{a}, \mathrm{b}}$ & 0.16 \\
\hline Calcified HAVICs & $5.65^{\mathrm{a}}$ & 0.35 & $6.85^{\mathrm{a}, \mathrm{b}}$ & 0.28 \\
\hline BAVICs & 56.78 & 7.16 & $81.32^{\mathrm{b}}$ & 10.39 \\
\hline
\end{tabular}

Values are represented as U/mg. DMEM = Dulbecco's Modified Eagle's Medium $\mathrm{OSM}=$ osteogenic media; $\mathrm{U}=$ units. ${ }^{\mathrm{a}} \mathrm{P}<0.05$ compared to BAVICs; ${ }^{\mathrm{b}} \mathrm{P}<0.05$ compared to DMEM

Table 1: ALP activity in HAVICs and BAVICs cultured in OSM.

\section{A}
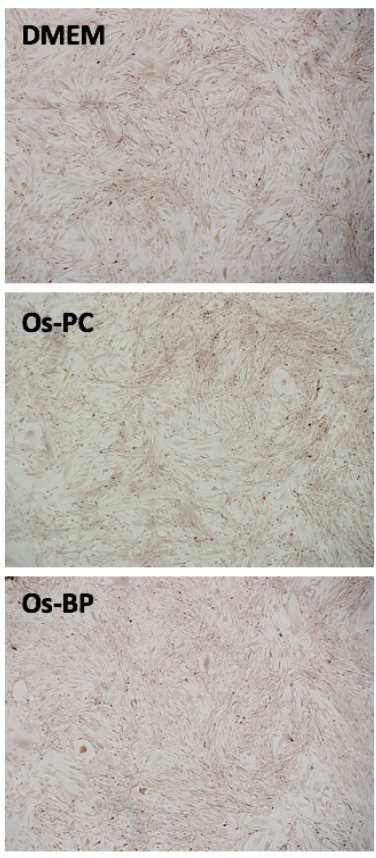

B

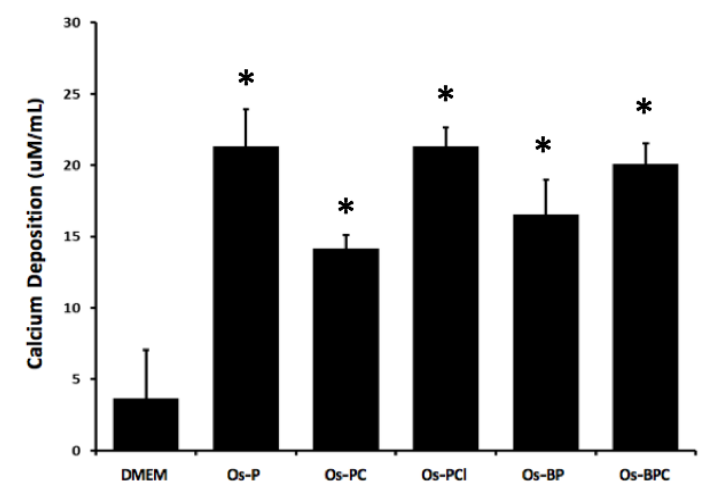

Figure 1: Calcification of HAVICs in different osteogenic media for 7 days (A) AR-S staining of HAVICs cultured in different osteogenic media. (B) Quantification of AR-S staining in BAVICs in different osteogenic media ${ }^{*} \mathrm{P}<0.05$. 
A
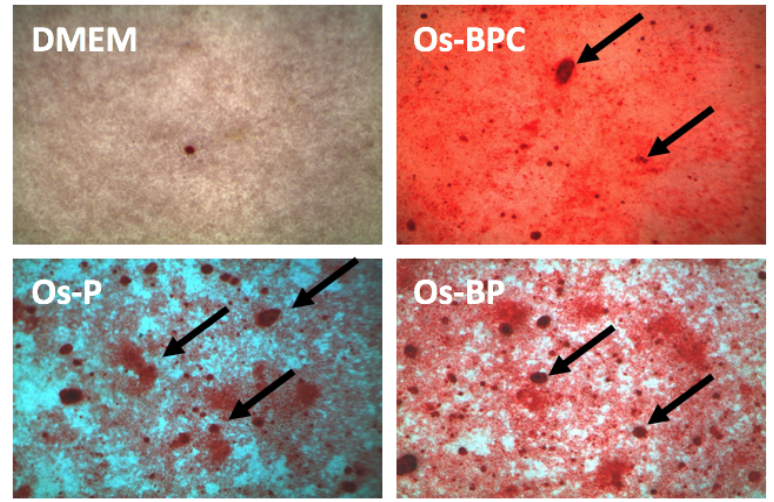

B

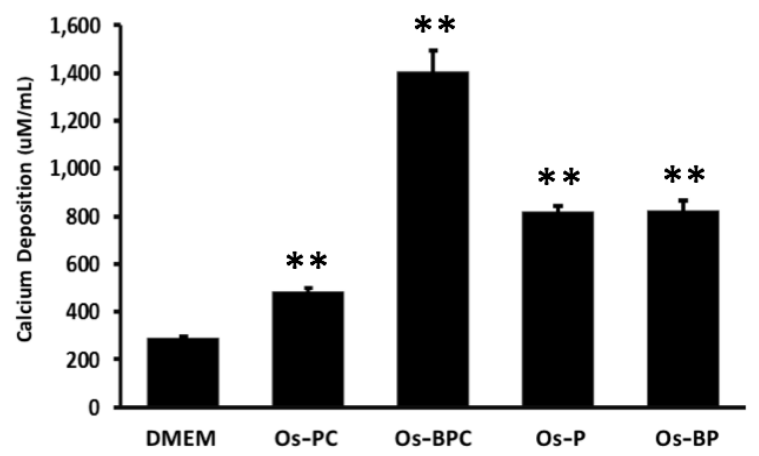

C

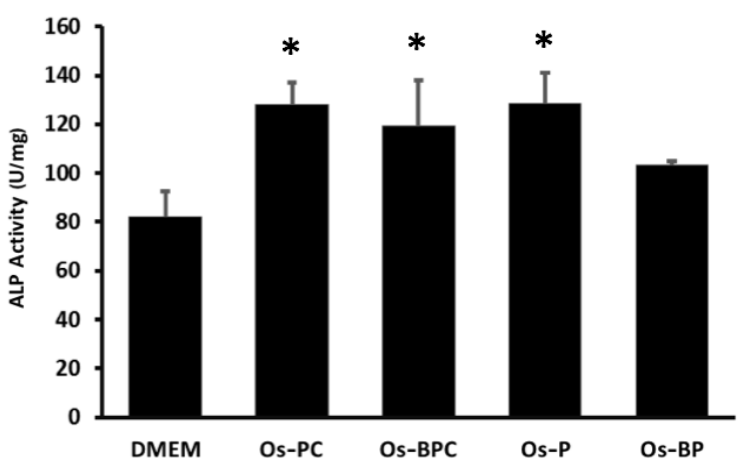

Figure 2: Calcification of BAVICs in different osteogenic media for 12 days (A) AR-S staining in BAVICs cultured in different osteogenic media. (B) Quantification of AR-S staining in BAVICs using different osteogenic media. (C) ALP activity in BAVICs cultured in different osteogenic media.

${ }^{*} \mathrm{P}<0.05 ;{ }^{* *} \mathrm{P}<0.0001$

among the 4 different OSM tested. The extent of calcification seen in BAVICs was significantly greater than that seen in HAVICs $(\mathrm{P}<0.0001)$. Interestingly, the extent of ALP activity increases did not match the extent of calcification seen with the 4 different OSM used here (Figure 2C), indicating that other factors besides ALP are involved in aortic cell calcification.

\section{ALP activity after treatment with EDTA in HAVICs}

There was a significant dose-dependent decrease in intracellular and extracellular ALP activity in HAVICs after treatment with $0.3 \mathrm{mM}$, $3 \mathrm{mM}$, and $6 \mathrm{mM}$ EDTA compared to DMEM controls $(\mathrm{P}<0.0001)$ (Figure 3). Treatment of HAVICs with $50 \mathrm{ug} / \mathrm{mL} \mathrm{Lp}(\mathrm{a})$ in the presence of with both $6 \mathrm{mM}$ EDTA significantly increased ALP activity compared to treatment with only $6 \mathrm{mM}$ EDTA $(\mathrm{P}<0.0001)$.

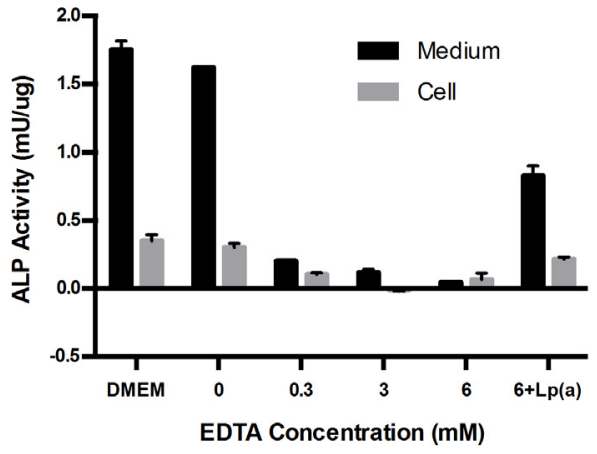

Figure 3: ALP activity in HAVICs after treatment with EDTA and Lp(a) for 7 days.

"P $<0.0001$ compared to DMEM; " $P<0.0001$ compared to $6 \mathrm{mM}$ EDTA in DMEM.

\section{Cellular proliferation and calcium deposition after treatment with the tankyrase 1/2 inhibitor XAV939}

HAVICs treated with $1 \mu \mathrm{M}, 5 \mu \mathrm{M}, 10 \mu \mathrm{M}$ and $20 \mu \mathrm{M}$ XAV939 resulted in significant reductions in cellular proliferation compared controls (Figure 4A). Subsequent experiments treating HAVICs with $2 \mu \mathrm{M}$ XAV939 showed a significant decrease in calcium deposition $(\mathrm{P}<0.05)$ (Figure $4 \mathrm{~B}$ and $\mathrm{C}$ ) and cellular proliferation $(\mathrm{P}<0.05)$ (Figure $4 \mathrm{E})$. However, when normalized to total protein concentration, calcium deposition was significantly increased (Figure 4D).

\section{Discussion}

The molecular mechanisms that contribute to AVS are not well understood and it is likely that multiple signaling pathways contribute to disease onset $[1,6]$. In this study, we examined ALP-dependent calcification in aortic valve interstitial cells from human and bovine models. There were significant differences in ALP activity between HAVICs and BAVICs cultured in DMEM and OSM, with BAVICs having the greatest ALP activity and degree of calcification. To further our analysis, we treated HAVICs with different concentrations of EDTA, a calcification reverser, and $\mathrm{Lp}(\mathrm{a})$, a calcification inducer [16]. There was a significant reduction in ALP activity in EDTA-treated HAVICs. The addition of $50 \mathrm{ug} / \mathrm{mL}$ of $\mathrm{Lp}$ (a) reversed the effects of EDTA in these cells. HAVICs treated with $2 \mu \mathrm{M}$ XAV939 significantly increased cellular proliferation and decreased calcium deposition. However, when normalized to total protein concentration, calcium deposition was significantly increased. We also found no change in calcification in porcine aortic valve interstitial cells when cultured in Os-P media (data not shown).

The process of aortic valve cell calcification is thought to involve multiple mechanisms and pathways, one of which is through ALP [1719]. ALPs are membrane-bound metalloenzymes suggested to play an active role in calcium deposition by decreasing levels of pyrophosphate, a calcification inhibitor ${ }^{8}$, and increasing levels of inorganic phosphate, which promotes mineralization [20]. ALP is highly expressed in human AVS through a complex cascade involving proinflammatory cytokines and factors [20-22] and is thought to play a prominent role in calcium deposition [21]. Furthermore, calcification inhibitors such as dynamin-related protein 1 [17], benzofuran [23], and XAV939 [24], reduce calcium deposition and are correlated with reductions in ALP activity. Therefore, it is likely that ALP plays a critical role during calcification in AVS. 
A

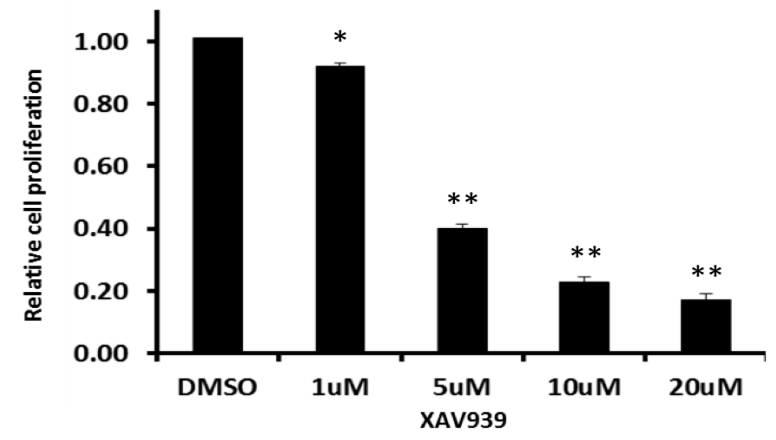

B

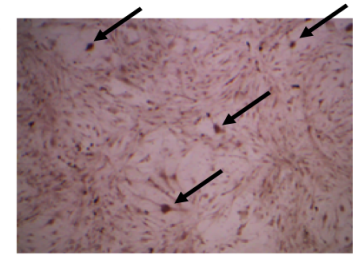

OSM

C

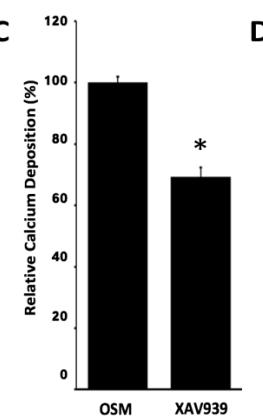

D $\frac{8}{8}$

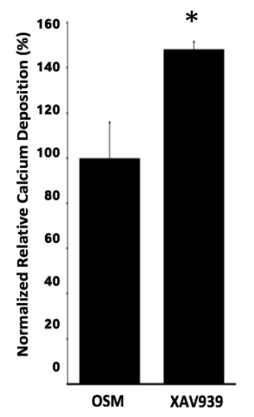

XAV939

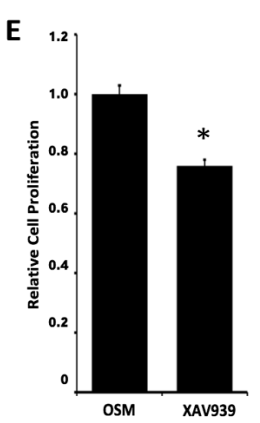

Figure 4: Cell proliferation and calcification of HAVICs after treatment with XAV939 for 21 days. (A) Relative cellular proliferation of HAVICs treated with 1-20 $\mu \mathrm{M}$ XAV939 for 48 hours. (B) AR-S staining of HAVICs treated with $2 \mu \mathrm{M}$ XAV939 for 21 days. (C) Quantification of AR-S staining of HAVICS treated with $2 \mu \mathrm{M}$ XAV939 for 21 days. (D) Quantification of AR-S staining of HAVICS treated with $2 \mu \mathrm{M}$ XAV939 for 21 days with protein normalization.

Previous studies showed that treatment of human mesenchymal stromal cells with XAV939 significantly decreased ALP activity [24]. Moreover, XAV939 induced cell apoptosis in neuroblastoma cells [25]. Here, we treated HAVICs with XAV939 and found that the latter dose-dependently decreased the number of HAVICs. At the same time, there appeared to be a significant reduction in calcification, however the contrary was seen when the amount of calcium was normalized to total protein measurement. It is possible that the concentration $(2 \mathrm{uM})$ used in this study is high and needed to be tittered, or the compound is simply toxic. Therefore, caution needs to be taken when using this molecule to inhibit calcification in HAVICs or BAVICs.

In this study, ALP activity and subsequent calcium deposition was found to be dependent on the levels of EDTA present in the media. Several studies have elucidated that EDTA plays a significant role as a calcification inhibitor [26-28]. Therefore, when studying aortic valve interstitial cell calcification, special attention needs to be paid to the content of EDTA in media and reagents used in the in vitro cell growth and calcification analyses. Furthermore, our group has recently demonstrated that $\mathrm{Lp}(\mathrm{a})$, known to be associated with AVS, significantly increased ALP and calcification of HAVICs [16]. Here, we show that Lp(a) significantly reversed EDTA's effect on HAVICs calcification.

The implications of our findings are quite relevant and applicable. In the past, different animals were used to study disease pathologies due to the limitations of acquiring human samples. However, we now have the means to study calcification in AVS using human tissues [14,15,29], which according to our results, may be the only effective way to study the disease. Our study is limited, however, in that it only explores ALP-dependent calcification and does not address other molecular contributors including Runx2 [30], Wnts [4], UII [15], BMPs [31], and TGF- $\beta$ [32]. Furthermore, we cultured our cells in OSM for 7 days for HAVICs and 12 days for BAVICs, while other studies assess calcification onset after much longer incubation times [33].

As we have found in our study, drawing comparative conclusions about AVS pathogenesis in humans is confounded when using bovine samples and particular OSM. We strongly suggest that future experiments attempting to understand the pathogenesis of AVS focus solely on aortic valve interstitial cells from human samples, as making comparison with other models may not be plausible. Thus, we advise careful consideration when making parallels between bovine and human samples in the assessment of aortic valve calcification.

\section{Acknowledgement}

This work was supported by the Canadian Institute of Health Research and NSERC.

\section{References}

1. Rajamannan NM, Evans FJ, Aikawa E (2011) Calcific aortic valve disease: Not simply a degenerative process: A review and agenda for research from the national heart and lung and blood institute aortic stenosis working group. Circulation 124: 1783-1791.

2. American Heart Association (2009) Aortic Valve Stenosis.

3. Mathieu P, Boulanger MC, Bouchareb R (2014) Molecular biology of calcific aortic valve disease: towards new pharmacological therapies. Expert Rev Cardiovasc Ther 12: 851-862.

4. Albanese I, Yu B, Al-Kindi H (2017) Role of Noncanonical Wnt Signaling Pathway in Human Aortic Valve Calcification. Arterioscler Thromb Vasc Biol 37: 543-552

5. Li C, Xu S, Gotlieb Al (2013) The progression of calcific aortic valve disease through injury, cell dysfunction, and disruptive biologic and physical force feedback loops. Cardiovasc Pathol 22: 1-8.

6. Lerman DA, Prasad S, Alotti N (2016) Calcific Aortic Valve Disease : Molecular Mechanisms and Therapeutic Approaches. Anatomy and Histology 10: 108112.

7. Shanahan CM, Crouthamel MH, Kapustin A, Giachelli CM (2012) Arterial Calcification in Chronic Kidney Disease: Key Roles for Calcium and Phosphate. Circ Res 109: 697-711.

8. Demer LL, Tintut $Y$ (2014) Inflammatory, metabolic, and genetic mechanisms of vascular calcification. Arter Thromb Vasc Biol 34: 715-723.

9. Villa-Bellosta R, Wang X, Millán JL, Dubyak GR, O’Neill WC (2011) Extracellular pyrophosphate metabolism and calcification in vascular smooth muscle. Am J Physiol Heart Circ Physiol 301: 61-68.

10. Haarhaus M, Brandenburg V, Kalantar-Zadeh K, Stenvinkel P, Magnusson P (2017) Alkaline phosphatase: a novel treatment target for cardiovascular disease in CKD. Nat Rev Nephrol 13: 429-442.

11. Ferdous Z, Jo H, Nerem RM (2013) Strain magnitude-dependent calcific marker expression in valvular and vascular cells. Cells Tissues Organs 197: 372-383.

12. Renato M, Bertacco E, Franchin C, Arrigoni G, Rattazzi M (2013) Heart Proteomics 1005: 95-107.

13. Rattazzi M, Bertacco E, lop $L$ (2014) Extracellular pyrophosphate is reduced in aortic interstitial valve cells acquiring a calcifying profile: Implications for aortic valve calcification. Atherosclerosis 237: 568-576.

14. Al Kindi $\mathrm{H}$, Hafiane A, You Z (2014) Circulating levels of the vasoactive peptide urotensin II in patients with acute coronary syndrome and stable coronary artery disease. Peptides 55: 151-157. 
Citation: Khan K, Yu B, Al-Kindi H, Cecere R, Schwertani A (2017) Assessing Calcification Onset in Aortic Valve Interstitial Cells. Cardiovasc Pharm Open Access 6: 222. doi: 10.4172/2329-6607.1000222

15. Albanese I, Daskalopoulou SS, Yu B (2016) The urotensin II system and carotid atherosclerosis: A role in vascular calcification. Front Pharmacol 7: 149.

16. Yu B, Hafiane A, Thanassoulis G (2017) Lipoprotein(a) Induces Human Aortic Valve Interstitial Cell Calcification. JACC Basic Transl Sci 2: 358-371.

17. Rogers MA, Maldonado N, Hutcheson JD (2017) Dynamin-Related Protein 1 Inhibition Attenuates Cardiovascular Calcification in the Presence of Oxidative Stress. Circ Res 121: 220-233.

18. Li F, Song R, Ao L (2017) ADAMTS5 Deficiency in Calcified Aortic Valves Is Associated with Elevated Pro-Osteogenic Activity in Valvular Interstitial Cells. Arterioscler Thromb Vasc Biol 37: 1339-1351.

19. Bonetti A, Della MA, Contin M (2017) Survival-Related Autophagic Activity Versus Procalcific Death in Cultured Aortic Valve Interstitial Cells Treated With Critical Normophosphatemic-Like Phosphate Concentrations. J Histochem Cytochem 65: 125-138.

20. Golub EE, Harrison G, Taylor AG, Camper S, Shapiro IM (1992) The role of alkaline phosphatase in cartilage mineralization. Bone Miner 17: 273-278.

21. Mathieu P, Voisine P, Pépin A, Shetty R, Savard N, et al. (2005) Calcification of human valve interstitial cells is dependent on alkaline phosphatase activity. J Hear Valve Dis 14: 353-357.

22. Shioi A, Katagi M, Okuno Y (2002) Induction of Bone-Type Alkaline Phosphatase in Human Vascular Smooth Muscle Cells: Roles of Tumor Necrosis Factoralpha and Oncostatin M Derived From Macrophages. Circ Res 91: 9-16.

23. Marquès S, Buchet R, Popowycz F, Lemaire M, Mebarek S (2016) Synthesis of benzofuran derivatives as selective inhibitors of tissue-nonspecific alkaline phosphatase: Effects on cell toxicity and osteoblast-induced mineralization Bioorganic Med Chem Lett 26: 1457-1459.
24. Yamada A, Iwata T, Yamato M, Okano T, Izumi Y (2013) Diverse functions of secreted frizzled-related proteins in the osteoblastogenesis of human multipotent mesenchymal stromal cells. Biomaterials 34: 3270-3278.

25. Tian XH, Hou WJ, Fang Y (2013) XAV939, a tankyrase 1 inhibitior, promotes cell apoptosis in neuroblastoma cell lines by inhibiting Wnt/ $\beta$-catenin signaling pathway. J Exp Clin Cancer Res 32: p100.

26. Maniscalco BS, Taylor KA (2004) Calcification in coronary artery disease can be reversed by EDTA-tetracycline long-term chemotherapy. Pathophysiology 11: 95-101.

27. Nehir S, Karaca K, Yilmaz N (2006) Two-stage EDTA anti-calcifi cation method for bioprosthetic heart valve materials. Med Sci Monit 12: 33-39.

28. Lei Y, Grover A, Sinha A, Vyavahare N (2013) Efficacy of reversal of aortic calcification by chelating agents. Calcif Tissue Int 93: 426-435.

29. He C, Tang H, Mei Z (2017) Human interstitial cellular model in therapeutics of heart valve calcification. Amino Acids PP: 1-17.

30. Cui RR, Li SJ, Liu LJ (2012) MicroRNA-204 regulates vascular smooth muscle cell calcification in vitro and in vivo. Cardiovasc Res 96: 320-329.

31. Davidson MM, Nesti C, Palenzuela L (2005) Novel cell lines derived from adult human ventricular cardiomyocytes. J Mol Cell Cardiol 39: 133-147.

32. Jian B, Narula N, Li Q, lii ERM, Levy RJ (2003) Progression of Aortic Valve Stenosis : TGF- B1 is Present in Calcified Aortic Valve Cusps and. Ann Thorac Surg 75: 457-466.

33. Liu S, Cheng C (2015) HHS Public Access. Wiley Interdiscip Rev RNA 4: 547 566. 